Adam Pilarski ${ }^{1}$, Nicole Penn ${ }^{1}$, Sharenja Ratnakumar ${ }^{1}$, Richard D. Barker ${ }^{2}$ and Heather J. Milburn ${ }^{1,3}$

${ }^{1}$ King's College London School of Medicine, London, UK. ${ }^{2}$ Dept of Respiratory Medicine, King's College Hospital, London,

UK. ${ }^{3}$ Dept of Respiratory Medicine, Guy's and St Thomas' NHS Foundation Trust, London, UK.

Correspondence: Heather J. Milburn, Chest Clinic, Guy’s Hospital, Great Maze Pond, London, SE1 9RT, UK.

E-mail: heather.milburn@gstt.nhs.uk

Received: Jan 022015 | Accepted after revision: July 162016 | First published online: Oct 062016

Conflict of interest: None declared.

Acknowledgements: The authors would like to thank Frank Post (GU Medicine, King's College Hospital, London, UK), Jessie Randhawa (Respiratory Medicine, Guy's and St Thomas' NHS Foundation Trust, London UK) and Vanessa Kahr (Respiratory Medicine, King's College Hospital, London, UK) for their support in the early stages of the project.

This report was a retrospective analysis of routine data, in which no personal identifiers were retained, and collected as part of clinical service provision.

H.J. Milburn and R.D. Barker conceived the study. N. Penn, A. Pilarski, S. Ratnakumar and R.D. Barker collected the data. N. Penn carried out the statistical analysis. With guidance from H.J. Milburn, N. Penn and A. Pilarski wrote the initial manuscript. A. Pilarski revised the manuscript with guidance from H.J. Milburn. All authors contributed to the completed manuscript.

\title{
References
}

1 Sita-Lumsden A, Lapthorn G, Swaminathan R, et al. Reactivation of tuberculosis and vitamin D deficiency: the contribution of diet and exposure to sunlight. Thorax 2007; 62: 1003-1007.

2 Ustianowski A, Shaffer R, Collin S, et al. Prevalence and associations of vitamin D deficiency in foreign-born persons with tuberculosis in London. J Infect 2005; 50: 432-437.

3 Prietl B, Treiber G, Pieber TR, et al. Vitamin D and immune function. Nutrients 2013; 5: 2502-2521.

4 Coussens AK, Wilkinson RJ, Hanifa Y, et al. Vitamin D accelerates resolution of inflammatory responses during tuberculosis treatment. Proc Natl Acad Sci USA 2012; 109: 15449-15454.

5 Martineau AR, Timms PM, Bothamley GH, et al. High-dose vitamin $\mathrm{D}(3)$ during intensive-phase antimicrobial treatment of pulmonary tuberculosis: a double-blind randomised controlled trial. Lancet 2011; 377: 242-250.

6 Salahuddin N, Ali F, Hasan Z, et al. Vitamin D accelerates clinical recovery from tuberculosis: results of the SUCCINCT Study [Supplementary Cholecalciferol in recovery from tuberculosis]. A randomized, placebocontrolled, clinical trial of vitamin D supplementation in patients with pulmonary tuberculosis. BMC Infect Dis 2013; 13: 22.

7 Pareek M, Innes J, Sridhar S, et al. Vitamin D deficiency and TB disease phenotype. Thorax 2015; 70: 1171-1180.

8 Pareek M, Evans J, Innes J, et al. Ethnicity and mycobacterial lineage as determinants of tuberculosis disease phenotype. Thorax 2013; 68: 221-229.

9 Coussens AK, Wilkinson RJ, Nikolayevskyy V, et al. Ethnic variation in inflammatory profile in tuberculosis. PLoS Pathog 2013; 9: e1003468.

10 Martineau AR, Leandro AC, Anderson ST, et al. Association between Gc genotype and susceptibility to TB is dependent on vitamin D status. Eur Respir J 2010; 35: 1106-1112.

11 Libon F, Cavalier E, Nikkels AF. Skin colour is relevant to vitamin D synthesis. Dermatology 2013; 227: 250-254.

12 Bogh MK, Schmedes AV, Philipsen PA, et al. Vitamin D production after UVB exposure depends on baseline vitamin D and total cholesterol but not on skin pigmentation. J Invest Dermatol 2010; 130: 546-553.

13 Gibney KB, MacGregor L, Leder K, et al. Vitamin D deficiency is associated with tuberculosis and latent tuberculosis infection in immigrants from sub-Saharan Africa. Clin Infect Dis 2008; 46: 443-446.

14 Talat N, Perry S, Parsonnet J, et al. Vitamin D deficiency and tuberculosis progression. Emerg Infect Dis 2010; 16: 853-855.

15 Lönnroth K, Migliori GB, Abubakar I, et al. Towards tuberculosis elimination: an action framework for low-incidence countries. Eur Respir J 2015; 45: 928-952.

\section{Association of pulmonary alveolar proteinosis and fibrosis: patient with GATA2 deficiency}

To the Editor:

Pulmonary alveolar proteinosis (PAP) is a rare syndrome characterised by an abnormal accumulation of surfactant proteins in pulmonary alveoli which impairs gas exchange [1]. PAP is classified into three groups: 
autoimmune PAP (aPAP), defined by the presence of anti-granulocyte-macrophage colony-stimulating factor (GM-CSF) autoantibodies, secondary PAP (sPAP) and genetic PAP. Haematological diseases are the most common causes of sPAP, particularly myelodysplastic syndrome (MDS) $[1,2]$. The diagnosis of PAP is based on a chest computed tomography (CT) scan, which demonstrates a crazy-paving pattern, and a typical positive periodic acid-Schiff (PAS) staining of bronchoalveolar lavage (BAL) [3]. Nowadays, a surgical lung biopsy is seldom needed to diagnose PAP, except for atypical presentation as in the case described below with concomitant pulmonary fibrosis and sPAP in a patient with GATA2 (GATA binding protein 2) deficiency.

A 28-year-old Caucasian woman from North Africa, a nonsmoker, an executive in a fuel company, was admitted to the Respiratory Dept of Pontchaillou Hospital (Rennes, France) in November 2013 with increasing New York Heart Association (NYHA) stage 2 dyspnoea, dry cough and interstitial lung disease. She had no significant family history and no past medical history except for right lower limb idiopathic lymphoedema diagnosed 5 years earlier (figure 1a). Digital clubbing was found on clinical examination as well as a normal chest auscultation. Retractile reticular opacities in both lung apices were noted on chest radiography. A chest CT scan showed diffuse bilateral thickening of the interlobular and intralobular septae predominant in the upper lobes, associated with profuse subpleural reticulations (figure 1b). Spirometry demonstrated a pure restrictive pattern: total lung capacity $68 \%$ of predicted and forced vital capacity (FVC) $52 \%$ of predicted. The transfer factor of the lung for carbon monoxide (TLCO) was reduced ( $40 \%$ of predicted). BAL was opalescent and found to contain 50000 cells $\mathrm{mL}^{-1}$ with $72 \%$ macrophages and $26 \%$ lymphocytes. PAS staining could not be performed on the BAL and a surgical lung biopsy was completed. Histological findings demonstrated a markedly thickened visceral pleura, and prominent subpleural and paraseptal fibrosis. Interstitial fibrosis accompanied alveoli filled with an eosinophilic, proteinaceous material which proved to be PAS-positive and diastase-resistant. Vacuolated, foamy alveolar macrophages and cholesterol crystals were also common. Rare fibroblastic foci and mild chronic inflammation were noted. Honeycombing, lymphoid aggregates, bronchiolisation, smooth muscle hyperplasia, squamous metaplasia, fibroelastosis and granulomas were absent (figure 1c). No anti-GM-CSF antibodies were identified in the sera. Haematological sPAP was suspected due to cytopenias: haemoglobin $10 \mathrm{~g} \cdot \mathrm{dL}^{-1}$, platelets $50 \mathrm{~g} \cdot \mathrm{L}^{-1}$, leukocytes $3.9 \mathrm{~g} \cdot \mathrm{L}^{-1}$, neutrophils $3.34 \mathrm{~g} \cdot \mathrm{L}^{-1}$, eosinophils $0.04 \mathrm{~g} \cdot \mathrm{L}^{-1}$, basophils $0.15 \mathrm{~g} \cdot \mathrm{L}^{-1}$, lymphocytes $0.33 \mathrm{~g} \cdot \mathrm{L}^{-1}$ and monocytes $0.04 \mathrm{~g} \cdot \mathrm{L}^{-1}$. Lymphocyte phenotype showed global B, T and natural killer lymphopenia. Gammaglobulins were normal: $12.4 \mathrm{~g} \cdot \mathrm{L}^{-1}$. A MDS was confirmed with a bone marrow biopsy (multilineage dysplasia and $<5 \%$ blasts with normal karyotype). Due to the association of the young age of the patient, lymphoedema, MDS and sPAP, GATA2 deficiency was looked for and was positive: exon 5 C.1020_1029dup: p.R344GfsX43. Over a 9-month period, the MDS remained stable although her dyspnoea symptoms deteriorated markedly, reaching NYHA stage 3 dyspnoea with a decrease in her pulmonary function tests (FVC dropping from $52 \%$ to $40 \%$ and TLCO dropping from $40 \%$ to $25 \%$ of the predicted values). Her chest radiograph, however, remained unchanged. A bilateral whole-lung lavage was performed in September 2014 without any effect on her respiratory symptoms, spirometry or chest CT scan. This led us to consider haematopoietic stem cell transplantation (HSCT). Her brother was HLA-matched and GATA2 deficiency was negative.

To the best of our knowledge, this is a novel frameshift GATA2 mutation and one of the rare descriptions of concomitant association of severe interstitial lung fibrosis with PAP in GATA2 deficiency at diagnosis [4]. It may be the end result of progressive chronic PAP or may be specific to PAP in patients with GATA2 deficiency. MARCHAND-ADAm et al. [5] described a sequential case of a 35-year-old man with PAP secondary to idiopathic bone marrow aplasia, followed 10 years later by idiopathic pulmonary fibrosis associated with heterozygous deleterious germline TERT (telomerase reverse transcriptase) mutation. Similarly, aPAP was diagnosed in a 63-year-old woman that evolved into pulmonary fibrosis 7 years later [6]. The crazy-paving pattern was replaced by diffuse fibrotic changes, with a subpleural honeycombing pattern. The findings may suggest a surfactant dysfunction in the pathogenesis of fibrotic lung disease [6]. In the literature, the diagnosis, management and prognosis of sPAP differ from other types of PAP. The presentation on chest CT scan is more atypical and the prognosis of sPAP is poorer than aPAP (2-year survival of $46 \%$ versus $100 \%$ ) $[2,3,7,8]$. The management of sPAP is often based on the treatment of the underlying disease $[1,2]$. Chemotherapy and early HSCT can cure sPAP. Whole-lung lavage is also an effective treatment to improve patient symptoms $[1,2,4]$.

In this case report, even at a Competence Centre for Rare Pulmonary Diseases, pulmonologists and radiologists did not consider the PAP diagnosis in the first instance. The Respiratory Dept at Pontchaillou Hospital is experienced in managing PAP, with more than 60 whole-lung lavages performed in patients with aPAP in the last 15 years $[1,9]$. PAS staining could not be performed on BAL afterwards. Transbronchial biopsies and especially cryobiopsies may be reliable diagnostic tools in interstitial lung diseases. However, thrombocytopenia led us to perform surgical lung biopsy, taking one single risk with easier bleeding management, in view of this atypical chest CT scan presentation. Indeed, the thickening of the septae in the upper lobes matching with the crazy-paving pattern, associated with profuse subpleural reticulations leading us 

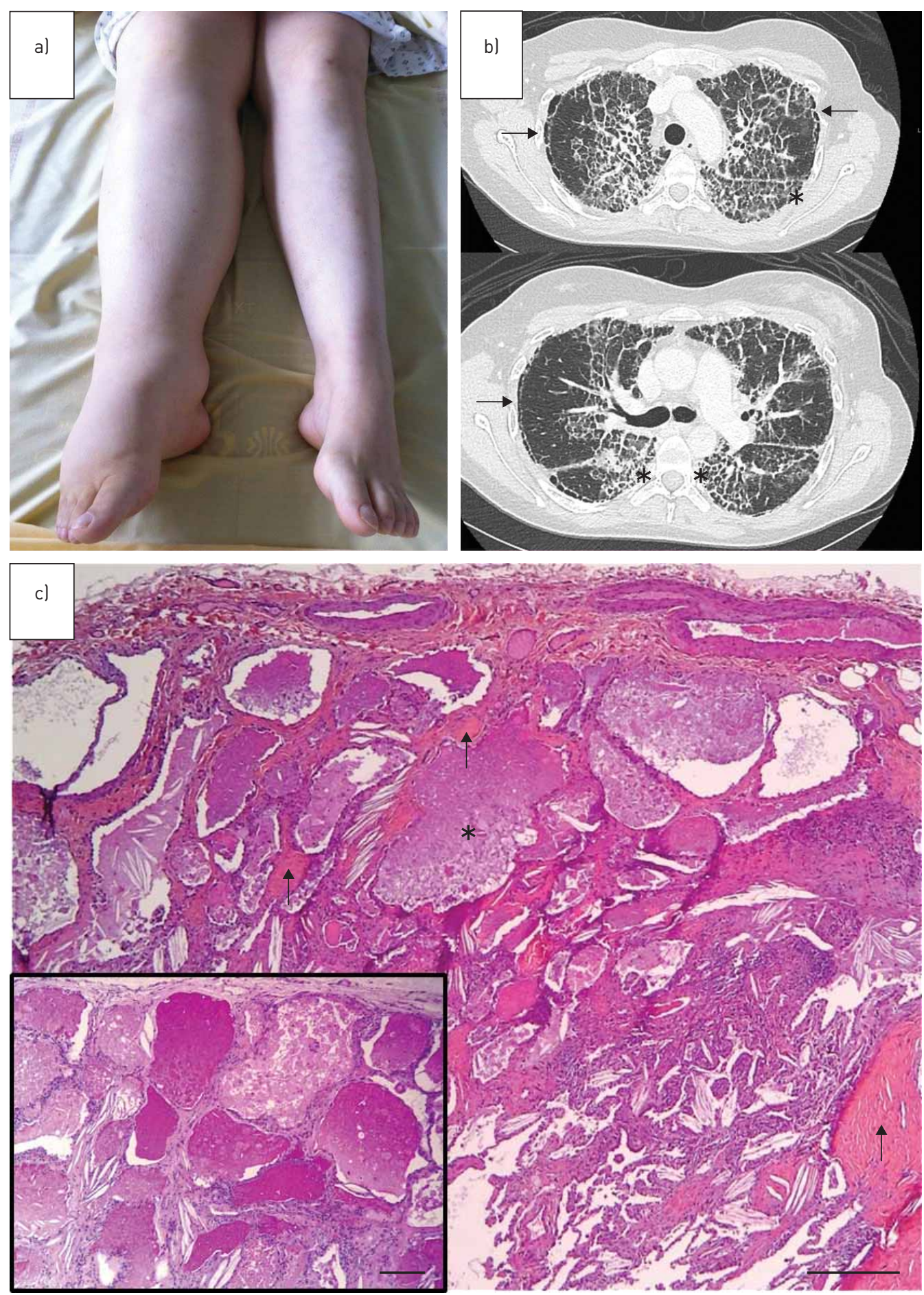

FIGURE 1 a) Right limb lymphoedema. b) Chest computed tomography image showing diffuse, bilateral and irregular thickening of the interlobular and intralobular septae clearly predominant in the upper lobes and apical segments of the lower lobes (asterisks). In the subpleural region of the upper lobes, we observed paraseptal emphysema (arrows). c) Surgical lung biopsy. Note the eosinophilic, granular, proteinaceous material within alveolar spaces characteristic of pulmonary alveolar proteinosis (asterisk). This material is periodic acid-Schiff (PAS)-positive (inset). Associated interstitial fibrosis is shown by arrows. Haematoxylineosin-safran: scale bar $=500 \mu \mathrm{m}$; PAS (inset): scale bar $=250 \mu \mathrm{m}$.

to fibrosis, were unexpected findings. Compared with aPAP, sPAP is more difficult to diagnose, not only due to the atypical chest CT scan findings, but also because of the absence of anti-GM-CSF antibodies and the possible underlying diseases. The presentation is more diffuse with less geographical pattern in sPAP than in aPAP: $62 \%$ versus $19 \%$ and $24 \%$ versus $71 \%$, respectively [8]. The subpleural ground-glass opacities and 
crazy-paving appearance are significantly less frequent in sPAP [8]. The use of surgical lung biopsy is more frequent in sPAP than aPAP (41\% versus 7\%) [7]. Thus, with an unclear interstitial lung disease, a PAS stain would be an interesting test to perform on BAL to avoid surgical lung biopsy.

In the cohort of IsHII et al. [2] of 31 patients with SPAP associated with MDS, the mortality rate was mainly due to the progression of PAP. Infections and the progression of the MDS were the second and third causes of mortality, respectively [2]. Only 10 out of 31 patients with MDS were treated with whole-lung lavage and only three had a positive response to the therapy [2].

GATA2 belongs to a family of zinc finger transcription factors that are critical regulators of gene expression in haematopoietic cells and GATA2 deficiency is a recent discovery [10]. GATA2 has been shown to regulate alveolar macrophage phagocytosis. In GATA2 deficiency, an alveolar macrophage dysfunction is present rather than a quantitative deficit given the abundance of alveolar macrophages in BAL fluid from patients affected by PAP [10].

SPINNER et al. [10] described the clinical features of 57 patients with proven mutations leading to GATA2 deficiency. They identified a broad phenotype encompassing immunodeficiency, haematologic disorder, PAP and lymphatic dysfunction, which match with our patient. $54 \%$ were female with a median (range) age at first visit of 30 (4-76) years and median (range) age at initial presentation of 20 years (5 months to 78 years). Of the 50 patients who underwent bone marrow biopsies, 42 patients (84\%) met diagnostic criteria for MDS with most bone marrow biopsies demonstrating multilineage dysplasia and $<5 \%$ blasts. Chronic lymphoedema was present in six patients (11\%). Biopsy-proven PAP was identified in 10 adults (18\%) [10]. In the Discussion section of SPINNER et al. [10], the authors report that the high prevalence of diffusion and ventilatory defects in their cohort may reflect alveolar filling, emphysematous changes, fibrosis or bronchiectasis secondary to recurrent pulmonary infection. However, no specific description of pulmonary fibrosis associated concomitantly with PAP has been described. The overall survival rate was $96 \%$ by age 20 years, $77 \%$ by age 40 years and $45 \%$ by age 60 years [10]. 21 patients underwent HSCT for MDS, PAP or recurrent infections. All patients with PAP demonstrated a significant improvement in pulmonary function $[10,11]$. Importantly, family members should be screened routinely for GATA2 mutations before donating their bone marrow. In this case report, HSCT is now considered. We are expecting a new pulmonary evaluation before allograft. The discussion is underway with the patient, her family, the pulmonologists and the current transplant team assessing the risk/benefit balance. Indeed, mortality risk increases with TLCO or forced expiratory volume in $1 \mathrm{~s} \leqslant 65 \%$ of predicted, NYHA stage 4 dyspnoea or oxygen therapy [1]. Otherwise, sPAP physiopathology supports a recurrence after lung transplantation because of alveolar macrophages quantitatively and qualitatively unable to provide surfactant clearance $[4,8]$.

In summary, this is one of the rare descriptions of concomitant association of severe pulmonary fibrosis and PAP at the diagnosis complicating a novel GATA2 mutation. This very rare syndrome is important to diagnose because of the discussion of HSCT before worsening of the respiratory status.

$\boldsymbol{0}$ @ERSpublications

Lymphoedema, myelodysplasia, fibrosis and pulmonary alveolar proteinosis complicating a novel GATA2 mutation http://ow.ly/RHci303h8ks

Alice Ballerie $\oplus^{1}$, Stanislas Nimubona ${ }^{2}$, Catherine Meunier ${ }^{3}$, Francisco Llamas Gutierrez ${ }^{4}$, Benoît Desrues ${ }^{1,5}$, Philippe Delaval ${ }^{1,6}$ and Stéphane Jouneau ${ }^{1,6}$

${ }^{1}$ Service de pneumologie, centre de compétences des maladies pulmonaires rares de Bretagne, hôpital Pontchaillou, Rennes, France. ${ }^{2}$ Service d'hématologie, hôpital Pontchaillou, Rennes, France. ${ }^{3}$ Service d'imagerie thoracique, hôpital Pontchaillou, Rennes, France. ${ }^{4}$ Service d'anatomie et cytologie pathologiques, hôpital Pontchaillou, Rennes, France.

${ }^{5}$ Université de Rennes 1, Rennes, France. ${ }^{6}$ IRSET UMR 1085, Université de Rennes 1, Rennes, France.

Correspondence: Alice Ballerie, Respiratory Dept, Pontchaillou Hospital, Rennes 1 University, 2 rue Henri Le Guilloux, 35033 Rennes Cedex, France. E-mail: alice.ballerie@chu-rennes.fr

Received: Feb 022016 | Accepted after revision: Aug 092016 | First published online: Oct 202016

Conflict of interest: None declared.

Acknowledgements: The authors thank Marie de Tayrac (Service de génétique moléculaire et génomique, hôpital Pontchaillou and UMR 6290 Institut de génétique et développement, Université Rennes 1, Rennes, France) for her help with molecular genetic databases.

\section{References}

1 Jouneau S, Kerjouan M, Briens E, et al. La proteinose alveolaire pulmonaire. [Pulmonary alveolar proteinosis.] Rev Mal Respir 2014; 31: 975-991. 
Ishii H, Seymour JF, Tazawa R, et al. Secondary pulmonary alveolar proteinosis complicating myelodysplastic syndrome results in worsening of prognosis: a retrospective cohort study in Japan. BMC Pulm Med 2014; $14: 37$.

3 Inoue $\mathrm{Y}$, Trapnell BC, Tazawa R, et al. Characteristics of a large cohort of patients with autoimmune pulmonary alveolar proteinosis in Japan. Am J Respir Crit Care Med 2008; 177: 752-762.

4 Griese M, Zarbock R, Costabel U, et al. GATA2 deficiency in children and adults with severe pulmonary alveolar proteinosis and hematologic disorders. BMC Pulm Med 2015; 15: 87.

5 Marchand-Adam S, Diot B, Magro P, et al. Pulmonary alveolar proteinosis revealing a telomerase disease. Am J Respir Crit Care Med 2013; 188: 402-404.

6 Luisetti M, Bruno P, Kadija Z, et al. Relationship between diffuse pulmonary fibrosis, alveolar proteinosis, and granulocyte-macrophage colony stimulating factor autoantibodies. Respir Care 2011; 56: 1608-1610.

7 Ishii $\mathrm{H}$, Tazawa $\mathrm{R}$, Kaneko $\mathrm{C}$, et al. Clinical features of secondary pulmonary alveolar proteinosis: pre-mortem cases in Japan. Eur Respir J 2011; 37: 465-468.

8 Ishii H, Trapnell BC, Tazawa R, et al. Comparative study of high-resolution CT findings between autoimmune and secondary pulmonary alveolar proteinosis. Chest 2009; 136: 1348-1355.

9 Briens E, Delaval P, Mairesse MP, et al. Lipoproteinose alveolaire pulmonaire. [Pulmonary alveolar proteinosis.] Rev Mal Respir 2002; 19: 166-182.

10 Spinner MA, Sanchez LA, Hsu AP, et al. GATA2 deficiency: a protean disorder of hematopoiesis, lymphatics, and immunity. Blood 2014; 123: 809-821.

11 Cuellar-Rodriguez J, Gea-Banacloche J, Freeman AF, et al. Successful allogeneic hematopoietic stem cell transplantation for GATA2 deficiency. Blood 2011; 118: 3715-3720.

\section{Bosutinib therapy resulting in severe deterioration of pre-existing pulmonary arterial hypertension}

To the Editor:

We read with interest the recent correspondence from QuiLOT et al. [1] describing a likely case of ponatinib-associated pulmonary arterial hypertension (PAH). The authors hypothesised that the mechanism by which tyrosine kinase inhibitors (TKIs) such as dasatinib and ponatinib, used for the treatment of chronic myeloid leukaemia $(\mathrm{CML})$, induce $\mathrm{PAH}$ may involve their common inhibition of the non-receptor tyrosine kinase, Src [1]. Here, we present a patient who developed marked worsening of pre-existing TKI-associated PAH following commencement of bosutinib, a third-generation TKI also known to inhibit Src [2]. After marked improvement on withdrawal of bosutinib, the patient experienced further significant worsening of PAH after commencing ponatinib. We believe this is the first reported case linking bosutinib with PAH. It also supports the association between ponatinib and PAH and represents the first time that the development or worsening of PAH associated with multiple TKIs in the same patient has been reported.

A 39-year-old man was diagnosed with CML in 2001. He had no other medical history of note. Following initial disease control with imatinib (March 2004-October 2009), he was switched to dasatinib because of loss of cytogenetic response. After 12 months of therapy, he became progressively breathless and developed pleural effusions. He was given diuretics and dasatinib was stopped in January 2011. Despite resolution of the pleural effusions and initial improvement in symptoms, he subsequently became more breathless and was assessed in our pulmonary hypertension (PH) unit in March 2011. Right heart catheterisation (RHC) confirmed severe PAH (mean right atrial pressure $15 \mathrm{mmHg}$; mean pulmonary arterial pressure $55 \mathrm{mmHg}$; pulmonary arterial wedge pressure $11 \mathrm{mmHg}$; cardiac index $2.5 \mathrm{~L} \cdot \mathrm{min}^{-1} \cdot \mathrm{m}^{-2}$; pulmonary vascular resistance 17.6 Wood units; and mixed venous oxygen saturation 58\%). Perfusion lung scanning excluded thromboembolic disease and there was no parenchymal lung disease or pleural effusions on computed tomography. His exercise capacity, as assessed by incremental shuttle walking distance (ISWD), was $180 \mathrm{~m}$ and he was in the World Health Organization (WHO) functional class (FC) III (figure 1). He was diagnosed with PAH associated with dasatinib therapy and commenced sildenafil $25 \mathrm{mg}$ three times a day.

By May 2011, he had demonstrated a good response to sildenafil with ISWD increasing to $680 \mathrm{~m}$ and his performance status had improved to WHO FC II. He subsequently commenced nilotinib $400 \mathrm{mg}$ twice a day in June 2011. In October 2011, he complained of some worsening of symptoms and sildenafil was increased to $50 \mathrm{mg}$ three times a day. His symptoms stabilised for the next 9 months, but in July 2012 he 